\title{
Determining crystal phase purity in $c$-BP through x-ray absorption spectroscopy
}

\author{
S. P. Huber, ${ }^{1,2, *}$ V. V. Medvedev,${ }^{3}$ E. Gullikson, ${ }^{4}$ B. Padavala, ${ }^{5}$ \\ J.H. Edgar, ${ }^{5}$ R. W. E. van de Kruijs, ${ }^{2}$ F. Bijkerk, ${ }^{2}$ and D. Prendergast ${ }^{1}$ \\ ${ }^{1}$ Molecular Foundry, Lawrence Berkeley National Laboratory, Berkeley, California 94720, United States \\ ${ }^{2}$ Industrial Focus Group XUV Optics, MESA+ Research Institute for Nanotechnology, \\ University of Twente, P.O. Box 217, 7500 AE, Enschede, The Netherlands \\ ${ }^{3}$ Institute for Spectroscopy RAS, Fizicheskaya str. 5, Troitsk, Moscow, 108840 Russia \\ ${ }^{4}$ Center for X-Ray Optics, Lawrence Berkeley National Laboratory, Berkeley, California 94720, United States \\ ${ }^{5}$ Department of Chemical Engineering, Kansas State University, Manhattan, Kansas 66506, United States
}

(Dated: Thursday $2^{\text {nd }}$ February, 2017)

\begin{abstract}
We employ x-ray absorption near-edge spectroscopy at the boron $K$-edge and phosphorus $L_{2,3^{-}}$ edge to study the structural properties of cubic boron phosphide (c-BP) samples. The x-ray absorption spectra are modeled from first-principles within the density functional theory framework using the excited electron core-hole $(\mathrm{XCH})$ approach. A simple structural model of a perfect $c$-BP crystal accurately reproduces the $\mathrm{P} L_{2,3}$-edge, however it fails to describe the broad and gradual onset of the B $K$-edge. Simulations of the spectroscopic signatures in boron $1 s$ excitations of intrinsic point defects and the hexagonal BP crystal phase, show that these additions to the structural model can not reproduce the broad pre-edge of the experimental spectrum. Calculated formation enthalpies show that, during the growth of $c$-BP, it is possible that amorphous boron phases can be grown in conjunction with the desired boron phosphide crystalline phase. In combination with experimental and theoretically obtained x-ray absorption spectra for an amorphous boron structure, which have a similar broad absorption onset in the B $K$-edge spectrum as the cubic boron phosphide samples, we provide evidence for the presence of amorphous boron clusters in the synthesized $c$-BP samples.
\end{abstract}

\section{INTRODUCTION}

The group III-V compound boron phosphide has been most commonly studied in its cubic zinc blende crystal phase $(c-\mathrm{BP})$, in which it is characterized as a refractory indirect gap semiconductor. Its high thermal conductivity $^{1}$, hardness, chemical stability, high neutron capture cross section ${ }^{1-3}$ and electronic structure, make it a promising candidate for application in catalysis ${ }^{4}$, hightemperature electronic devices ${ }^{5,6}$, neutron detectors ${ }^{7}$ and radiation protective coatings. Boron phosphide has also been suggested as a promising material for optical elements for extreme ultraviolet radiation, operating close to the $L_{2,3}$ absorption edge of the phosphorus ${ }^{8,9}$. Many studies have successfully grown this crystalline phase of boron phosphide; studies to further improve its structural quality are ongoing ${ }^{1-3,10-17}$.

For the majority of applications of cubic boron phosphide, the structural crystal quality is of great importance, as the useful properties of the materials are often negatively affected by the presence of structural imperfections. These imperfections can range from simple intrinsic crystal point defects, to agglomerations of structural defects and grain boundaries. In principle, it is also possible that other crystal phases, or even amorphous phases, grow alongside the intended phase. To improve the capabilities of current growth methods to grow high quality crystals, it is crucial to be able to analyze and characterize the structural properties of samples synthesized with these methods. In parallel with typical experimental techniques such as x-ray diffraction, to characterize the long range order found in the sample crystal structure, one could use methods that study the structural and chemical properties on a local atomic scale. This is possible with $\mathrm{X}$-ray absorption near-edge spectroscopy (XANES), as it is sensitive to structural point defects in the boron $K$-edge of various boron compounds ${ }^{18-20}$ and is typically highly sensitive to the different crystal phases of a particular compound. With the recent prediction ${ }^{21}$ of the hexagonal phase of boron phosphide $(h-\mathrm{BP})$, the subject of several recent studies ${ }^{22-27}$, XANES is a promising candidate as a method that will be able to determine the presence of this unintended crystal phase.

In this work, we present experimental x-ray absorption spectroscopy data, collected in the total electron yield (TEY) mode for both the B $K$-edge as well as the $\mathrm{P} L_{2,3}$-edge, of $c$-BP samples grown through chemical vapor deposition. To the best of the authors' knowledge, to this date neither the boron $K$-edge nor the $\mathrm{P}$ $L_{2,3}$ absorption edges have been reported in literature, either experimentally or theoretically. The experimentally collected spectra are simulated from first-principles within the density functional theory framework in order to model the structure of the grown $c$-BP samples. Our results show that a structural model defined by the perfect cubic crystal structure is able to accurately reproduce the $\mathrm{P} L_{2,3}$-edge but simultaneously fails to describe the B $K$-edge to the same degree of accuracy. We will discuss multiple refinements to the structural model that could hypothetically explain this juxtaposition, such as crystalline point defects and different crystal or amorphous phases. The results provide strong evidence for the presence of amorphous boron phases incorporated in the cubic boron phosphide crystal structure. 


\begin{tabular}{|c|c|c|c|c|c|c|}
\hline ID & $\mathrm{B}_{2} \mathrm{H}_{6}\left(1 \%\right.$ in $\left.\mathrm{H}_{2}\right)$ & $\mathrm{PH}_{3}$ & $\mathrm{H}_{2}$ & $p$ (torr) & $T\left({ }^{\circ} \mathrm{C}\right)$ & $t(\min )$ \\
\hline S1 & 30 & 80 & 4000 & 700 & 1200 & 30 \\
\hline S2 & 30 & 80 & 4000 & 700 & 800 & 5 \\
\hline
\end{tabular}

TABLE I. Experimental conditions during deposition of the two reference samples studied in this work, with partial pressures of precursor gases in sccm, total pressure $p$ in torr, temperature $T$ in ${ }^{\circ} \mathrm{C}$ and deposition time $t$ in $\min$.

\section{EXPERIMENTAL DETAILS}

In this section, we will report on the experimental details of sample deposition and the experimental x-ray absorption spectroscopy (XAS) measurements of the boron $K$-edge and phosphorus $L_{2,3}$-edge, performed at the Advanced Light Source (ALS). We also report on results obtained from standard thin film analyses, including $\mathrm{x}$ ray photoelectron spectroscopy (XPS), x-ray diffraction (XRD) and scanning electron microscopy (SEM), some of which can be found in the Supplementary Information.

\section{A. Cubic boron phosphide sample deposition}

The two cubic boron phosphide samples analyzed in this work were deposited onto $4 H$-SiC(0001) substrates miscut $4^{\circ}$ towards [1100], by chemical vapor deposition (CVD). The CVD setup and experimental methods used in this work have been described in greater detail elsewhere ${ }^{1,2,16}$. The precursor gases were ultra-high purity phosphine $(99.999 \%)$ and diborane $\left(1 \%\right.$ in $\left.\mathrm{H}_{2}\right)$ in an ultra-high purity hydrogen carrier gas. Detailed deposition conditions employed are listed in Table I. The deposition temperature was set to $1200 \mathrm{~K}$ for S1, the optimal temperature for crystal growth, and to a suboptimal temperature of $800 \mathrm{~K}$ for sample S2.

\section{B. Boron and boron nitride sample deposition}

To compare structural and x-ray absorption spectroscopy properties of the cubic boron phosphide samples, we also deposited boron and boron nitride samples as a reference. These reference samples were deposited in a ultra-high vacuum (UHV) chamber with base pressure of $1 \times 10^{-8}$ mbar, using electron-beam evaporation of a boron target onto the native oxide of single crystalline silicon substrates. The target thickness was approximately $40 \mathrm{~nm}$ for both samples, monitored by quartz mass balances, which typically yields an uncertainty of $10 \%$ in the final film thickness. For the growth of the boron nitride sample, a repetitive process was employed where first a thin layer of $0.7 \mathrm{~nm}$ boron was deposited, which was subsequently treated with nitrogen ion implantation for $240 \mathrm{~s}$ to create boron nitride. Nitrogen ions were produced with a Kaufman type hot cathode ion source oper- ated at $60 \mathrm{~mA}$ and $150 \mathrm{~V}$. Post-deposition XPS analysis of the boron nitride sample revealed that all the boron had reacted with the nitrogen and no elementary boron remained. The sample substrate holder remained near room temperature during the deposition process and the structure of the grown samples were determined to be amorphous by post deposition x-ray diffraction analysis.

\section{Total electron yield x-ray absorption spectroscopy}

All x-ray absorption spectroscopy measurements were carried out at beamline 6.3 .2 of the Advanced Light Source (ALS) synchrotron at Lawrence Berkeley National Laboratory (LBNL). A detailed description and characterization of the beamline and measurement chamber can be found elsewhere ${ }^{28,29}$. X-ray absorption measurements of the boron $K$-edge and phosphorus $L_{2,3}$ edge, were collected in TEY mode. The linearly polarized incident soft $\mathrm{x}$-ray beam was oriented at $1.5^{\circ}$ to the sample surface normal. Photon energies were calibrated by comparing to absolute absorption edges of $\mathrm{Si}$ and $\mathrm{B}$ filters installed at the beamline. For the monochromator, a grating with 200 lines/mm and an exit slit of $40 \mu \mathrm{m}$ was used, yielding an energy resolution $E / \Delta E$ of approximately 900 and 800 , at $130 \mathrm{eV}$ and $190 \mathrm{eV}$, respectively. This results in an approximate resolution of $0.14 \mathrm{eV}$ and $0.24 \mathrm{eV}$ at the $\mathrm{P} L_{2,3}$ and $\mathrm{B} K$-edge, respectively. The TEY current was measured as a function of incident photon energy in incremental steps of $0.1 \mathrm{eV}$ at both the $\mathrm{B} K$ and $\mathrm{P} L_{2,3}-$ edge. For each step, the average of 50 samples was taken for the final spectrum and the collection time was kept constant over all incident photon energies. The collected spectra have the dark current signal subtracted to account for the systematic error and noise in the collector electronics. Subsequently, the spectra are normalized by a spectrum collected by a photodiode to account for the intensity fluctuations in the x-ray beam as a function of photon energy.

\section{COMPUTATIONAL METHOD}

\section{A. X-ray absorption spectroscopy}

X-ray absorption spectroscopy was simulated within the density functional theory excited electron and corehole (DFT-XCH) approach ${ }^{30}$ where the photo excited atom is modeled by removing a core electron during the generation of the corresponding excited state pseudopotential and placing it in the first available empty state in subsequent valence electron calculations. The electronic structure problem, with this core-hole occupation constraint, is solved self-consistently. Non-selfconsistent band structures and associated eigenstates are generated efficiently for numerical convergence in Brillouin zone integrations using the Shirley interpolation 
scheme $^{31}$. Plane waves were expanded up to a maximum of $30 \mathrm{Ry}$ and ultrasoft pseuodpotentials ${ }^{32,33}$ were employed. The absorption spectrum is computed by evaluating the transition probability between the initial and final state as given by Fermi's golden rule within the dipole approximation. The resulting spectrum is broadened uniformly with a Gaussian of $0.2 \mathrm{eV}$ FWHM for all structural models. To correct for the well-known underestimation of the band gap and band width by local or semilocal functionals, such as the PBE functional, the energy scale is stretched uniformly by a factor of 1.08 for the phosphorus $L_{2,3}$-edge of all boron phosphide structures, both crystalline and amorphous. For the boron $K$-edge, in all boron and boron nitride structures, a stretching factor of 1.08 and 1.04 is used, respectively.

Due to the lack of an absolute energy reference inherent in the pseudopotential method, an energy alignment scheme was employed to yield comparably meaningful relative energies for structurally and chemically different systems $^{34}$. This approach does not necessarily yield a perfect relative alignment, as is the case for the systems studied here, which will be addressed in greater detail later in this work. Finally, the spectra were shifted by a single value, which was kept identical for all computed spectra of one particular compound, regardless of its crystal phase or structure. The compound specific value was obtained by comparing the computed x-ray absorption spectrum for the perfect crystal structure with the corresponding experimental spectrum. In the case of an $L_{2,3^{-}}$ edge transition, electrons from either the $2 p_{3 / 2}$ or $2 p_{1 / 2}$ core level can be excited, which, due to spin-orbit coupling, are energetically split. Under the assumption that excitations from $2 p_{3 / 2}$ or $2 p_{1 / 2}$ core levels are equally probable, in this work, this effect is accounted for by adding the spectrum to itself, after shifting it by an energy $\Delta E$ equal to the spin-orbit splitting of the relevant element, and properly weighting the two spectra by a ratio of $2: 1$ that reflects the population ratio of the $2 p_{3 / 2}$ and $2 p_{1 / 2}$ core levels.

In the case of $h$-BN, a non-isotropic material, the angle of incidence of the incident $\mathrm{x}$-ray beam with respect to the planar surface of the material has to be taken into account when modeling the x-ray absorption spectroscopy for a polarized light source. The final spectrum is modeled by taking a superposition of the in-plane and outof-plane contributions, weighted by the geometric factor defined by the dot product between the planar surface normal and the direction of the electromagnetic field.

\section{B. Structural optimizations}

All structural optimizations were performed within the density functional theory (DFT) framework as implemented in the Vienna $a b$ initio simulation package VASP $^{35}$. The DFT computations were performed using a generalized gradient approximation (GGA) functional parametrized by Perdew, Burke, and Ernzerhof (PBE) ${ }^{36}$ and ultrasoft pseudopotentials within the projector augmented wave (PAW) method $^{37,38}$, where the $2 s$ and $2 p$ electrons for boron and the $3 s$ and $3 p$ electrons for phosphorus are treated as valence electrons. Plane waves, to represent the wave functions, were expanded up to an energy of $400 \mathrm{eV}$ and the Brillouin zone was sampled at the $\Gamma$ point. The condition for structural relaxation was defined as a maximum Hellmann-Feynman force of $0.02 \mathrm{eV}^{-1}$ acting on any individual nucleus.

\section{Crystal cell definitions and molecular dynamics}

The crystalline $\alpha$-rhombohedral boron $(\alpha$-B), hexagonal boron nitride $(h$-BN), hexagonal boron phosphide $(h$-BP) and cubic boron phosphide $(c-\mathrm{BP})$ structures, were constructed as $2 \times 2 \times 2,4 \times 4 \times 2,4 \times 4 \times 2$ and $3 \times 3 \times 3$ supercells from the corresponding crystalline unit cell, respectively. The resulting $\alpha$-B, $h$-BN, $h$-BP and $c$-BP supercells had a total of 288, 128, 128 and 216 atoms, respectively. Finite temperature effects on the crystal structures were simulated by sampling the canonical ensemble $(N V T)$ at a temperature of $300 \mathrm{~K}$ regulated by a Nosé-Hoover thermostat. The time step of integration was set to 0.2 fs for all crystal structures. The systems were thermally equilibrated for at least $5 \mathrm{ps}$, after which the micro canonical ensemble $(N V E)$ was sampled, taking at least 2 snapshots, separated by at least 1 ps. The calculated x-ray absorption spectrum for each crystal structure is defined as the average of the spectra computed for each of the snapshots taken from the corresponding equilibrated molecular dynamics trajectory. For the planar hexagonal structures, van der Waals interactions were accounted for by applying the corrective scheme of Tkatchenko and Scheffler ${ }^{39}$ as implemented in VASP.

\section{Simulation of amorphous structures}

All amorphous structures discussed in this work were simulated using first-principles molecular dynamics methods implemented in the aforementioned software package VASP. The overall approach was identical for all generated amorphous networks and consisted largely of three consecutive steps. Firstly, the initial crystal structure was heated instantaneously to temperatures well above the experimental melting temperature of the respective crystal phase, by coupling the system to a NoséHoover thermostat. The $N V T$ ensemble was sampled at the elevated temperature until a structural defect arose that was strong enough to break the crystal symmetry and initiate the melting process of the crystal structure, which typically took between 200 fs to 4000 fs. The liquid was sampled at the elevated temperature for at least 3 ps to allow for significant atomic diffusion to occur, after which the temperature was gradually reduced to $300 \mathrm{~K}$ at a rate of approximately $1 \times 10^{-15} \mathrm{~K} \mathrm{~s}^{-1}$. After quench- 


\begin{tabular}{lccccc}
\hline ID & B \% & P \% & P(O) \% & C \% & O \% \\
\hline S1 & 39.0 & 34.3 & 3.4 & 14.4 & 9.0 \\
S2 & 39.6 & 38.3 & 4.2 & 6.8 & 11.1 \\
\hline
\end{tabular}

TABLE II. Elemental abundances for each of the two samples as determined from the experimental XPS analysis.

ing from the liquid, the amorphous structure was equilibrated at $300 \mathrm{~K}$ for at least $3 \mathrm{ps}$ before the trajectory was sampled for snapshots or structural properties. The initial crystal cells that were used for the simulation of the amorphous boron $(a-\mathrm{B})$, boron nitride $(a-\mathrm{BN})$ and boron phosphide $(a$-BP) structures, were the crystalline supercells described in the previous section, consisting of 288, 128 and 216 atoms, respectively.

\section{EXPERIMENTAL RESULTS}

\section{A. X-ray photoelectron spectroscopy}

The elemental composition of both samples was analyzed through XPS measurements performed with a Thermo Scientific Theta Probe instrument, employing monochromatic Al-K $\alpha$ radiation. Quantification of relative elemental abundances was realized employing tabulated sensitivity factors for Al- $K \alpha$ radiation $^{40}$. The binding energy of the boron $1 s$ core level overlaps with the phosphorus $2 s$ core level and therefore special care has to be taken when fitting XPS data to extract the elemental stoichiometry of the analyzed samples. The intensity ratio of the $\mathrm{P} 2 s$ signal with respect to the $\mathrm{P} 2 p_{3 / 2}$ signal was determined from a MoP reference sample that ostensibly contains phosphorus but no boron. This ratio was 1.15 and was kept constant during the subsequent XPS analysis of the $\mathrm{BP}$ samples. First, the $\mathrm{P} 2 p$ signal was fit to determine the total intensity of the elemental $\mathrm{P} 2 p$ core-level electrons, which in combination with the earlier determined $\mathrm{P} 2 s / \mathrm{P} 2 p_{3 / 2}$ intensity ratio, fixes the total expected intensity of the $\mathrm{P} 2 s$ signal. While keeping the peak area of the $\mathrm{P} 2 s$ component fixed, the combined $\mathrm{P} 2 s$ and $\mathrm{B} 1 s$ signal was fit into its individual components.

The result of the XPS data analysis is shown in Fig. 1. The left column in Fig. 1, shows the collected signal from electrons excited from P2 core-level electrons, and its decomposition in the various individual contributions. As described in the previous paragraph, the area of the $\mathrm{P} 2 p_{3 / 2}$ peak was subsequently used to fix the area of the $\mathrm{P} 2 s$ peak in the analysis of the $\mathrm{P} 2 s / \mathrm{B} 1 s$ signal, shown in the right column of Fig. 1, which clearly shows the overlapping signals of the $\mathrm{P} 2$ and $\mathrm{B} 1 s$ contributions.

From the area of the partial fit contributions, shown in Fig. 1, we can extract the abundance of the corresponding element. The results are shown in Table II.

Both samples have a non-negligible amount of oxygen and carbon, which is unsurprising given that the sam-

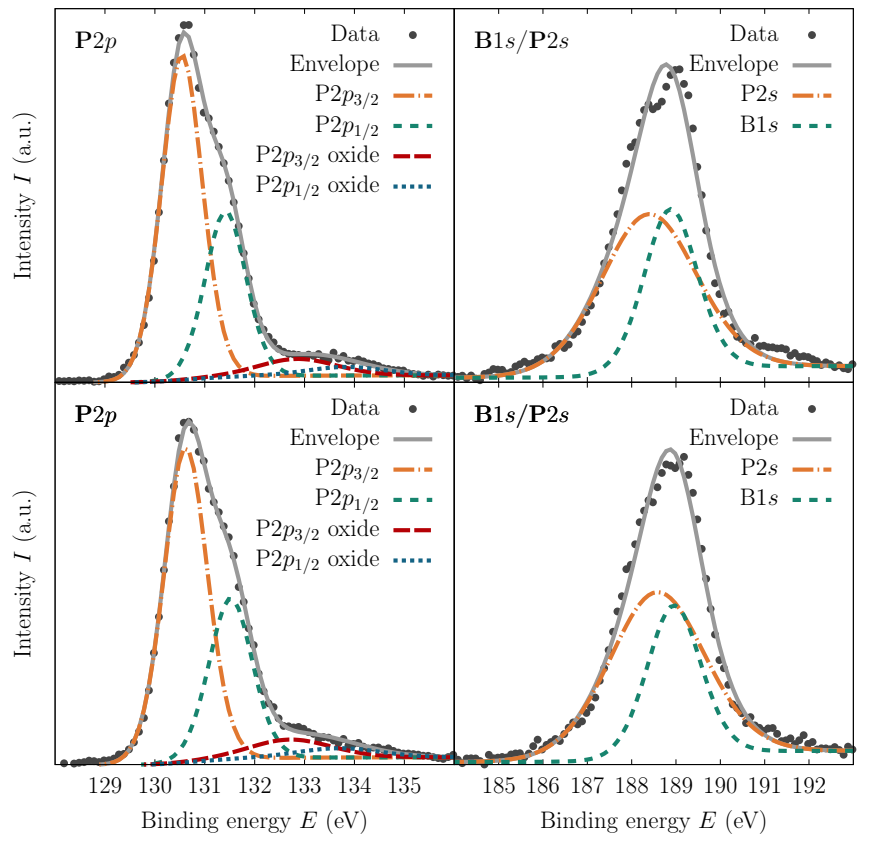

FIG. 1. In rows, from top to bottom, the measured and analyzed XPS data for samples S1 and S2, respectively. The columns, from left to right, represent the collected signal from excitations of electrons from $\mathrm{P} 2 p$ and $\mathrm{B} 1 s / \mathrm{P} 2 s$ core-levels, respectively. The experimental data is represented by black dots and the dashed colored lines represent the partial contributions to fit the experimental data. The solid gray line, labeled "Envelope", is the sum of these partial fit contributions.

ples have been exposed to ambient conditions between the deposition and XPS measurements. As demonstrated by the presence of oxide contributions in the $\mathrm{P} 2 p$ signal shown in Fig. 1, at least part of the oxygen is incorporated in the form of oxidized phosphorus. The carbon contribution most likely arises from carbohydrates that are adsorbed to the sample surface. The XPS analysis shows that sample $\mathrm{S} 1$ has a significantly larger abundance of $\mathrm{C}$ compared to $\mathrm{S} 2$, the reason for which is unclear. The abundances of boron and phosphorus for samples S1 and $\mathrm{S} 2$ are comparable, with a slightly higher phosphorus content for S2. Both samples appear to be off-stoichiometric with an excess of boron.

The samples have also been analyzed with XRD and SEM, details of which can be found in the Supplementary Information (SI). Both SEM and XRD reveal a strongly textured morphology of the samples (see Fig. S1 and Fig. S2 of the SI, respectively), which made it impossible to quantify the absolute or relative crystallinity of the thin films. Due to this limitation, it is not possible to definitively prove the presence of amorphous phases in the samples, however the data also does not pertinently exclude this possibility. The importance of the potential presence of amorphous phases in the grown thin films will become evident later in this work. 

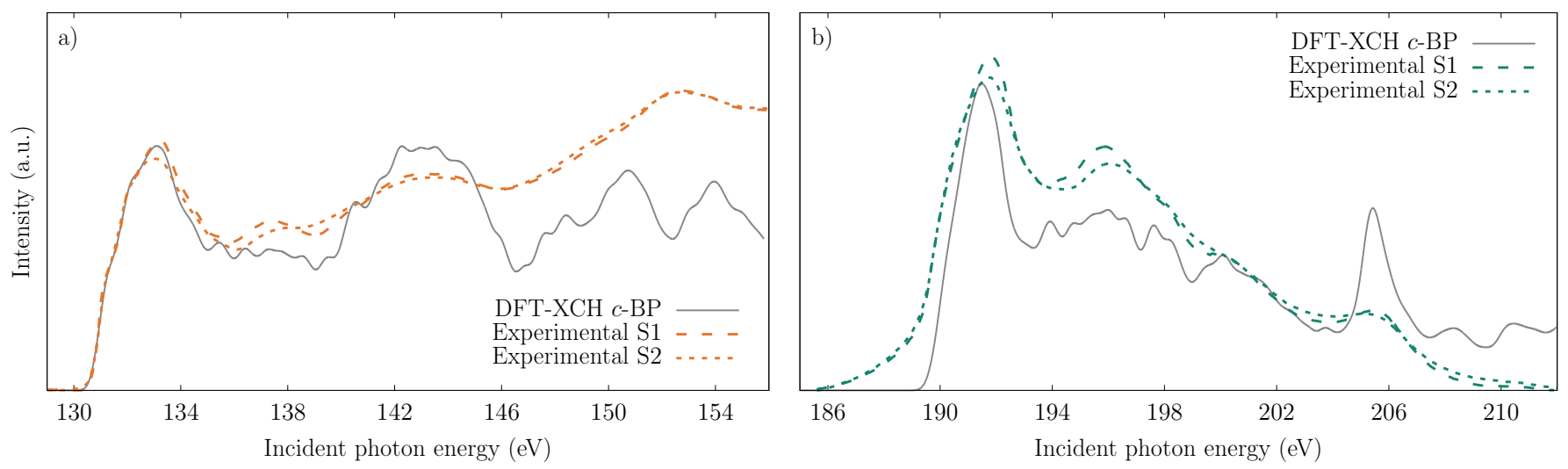

FIG. 2. The dashed colored lines represent the experimentally collected x-ray absorption spectra for the (a) phosphorus $L_{2,3}{ }^{-}$ edge and the (b) boron $K$-edge for the two reference $c$-BP samples, obtained in TEY mode. The solid black line in both panels corresponds to the simulated spectrum, computed with the DFT-XCH method for the perfect $c$-BP crystal structure, equilibrated at room temperature.

\section{B. X-ray absorption spectroscopy}

\section{COMPUTATIONAL RESULTS}

For each of the samples listed in Table I, the x-ray absorption spectrum, at both the boron $K$-edge and the phosphorus $L_{2,3}$-edge, has been collected in TEY mode, as shown in Fig. 2. The area under the curves has been normalized to unity to enable meaningful comparison of relative changes in intensity of certain features.

Despite the significant difference in experimental conditions, under which the two BP reference samples were deposited, specifically the difference in temperature, the collected spectra for both absorption edges are remarkably similar. The main discrepancy between the spectra of the two samples is the slight broadening of the spectral features for sample S2 compared to S1. Since XANES probes the chemical and structural environment in the direct vicinity of the excited atom, it is insensitive to long range amorphous or crystal structures. However, distortions of the perfect crystal lattice, as in for example amorphous phases, will also distort local structural properties, like bond angles and bond lengths. Distortions from the equilibrium structure on the local scale, induced by amorphization, are reflected in the x-ray absorption spectra by the broadening of the characteristic crystalline features. These broadening effects are partly due to new electronic transitions, induced by the structural defects of the crystal lattice, and are therefore not necessarily symmetrical or uniformly distributed, as would be the case in for example diffraction experiments. The broadening of features in the spectra for sample $\mathrm{S} 2$ can hence be attributed to the presence of amorphous phases, however it should be emphasized that the results show that the short range structural properties remain predominantly and distinctively cubic, as will be shown in the following section.
The solid black lines in Fig. 2 represent the simulated $\mathrm{x}$-ray absorption spectra for the perfect $c$-BP crystal thermalized at a temperature of $300 \mathrm{~K}$. Overall the simulated spectra agree well with experiment for both the B $K$-edge as well as the $\mathrm{P} L_{2,3}$ edge. The experimental features are generally smoother compared to the theory, which can be attributed to fact that the experimental samples are not perfect crystals and slight distortions from the perfect lattice lead to a broadening of the typical crystalline spectral features. The experimental energy resolution is with $\sim 0.2 \mathrm{eV}$ much narrower than the observed broadening and can therefore clearly not be the cause.

The DFT-XCH method used in the simulations, possesses the highest fidelity close to the absorption edge and will become less trustworthy for higher excitation energies. Discrepancies between experiment and theory close to the absorption edge are for that reason of most interest and therefore the most significant discrepancy in the data, is the absorption onset of the B $K$-edge. The theory models a sharp and sudden onset of the absorption edge, whereas the spectra collected for both samples S1 and S2, show a broad and gradual onset. In the following sections we will discuss several hypotheses that could potentially explain the discrepancy in the predicted and measured B $K$-edge, one of which is the presence of crystal point defects in the experimental samples.

\section{A. X-ray absorption spectroscopy of point defects}

X-ray absorption spectroscopy of the B $K$-edge is sensitive to point defects of the crystal structure in various boron compounds ${ }^{18-20,41-43}$. To determine whether point defects are capable of explaining the broad onset of absorption observed in experiment, we have computed 
the x-ray absorption spectra for several point defects. We consider boron antisite $\mathrm{B}_{\mathrm{P}}$, phosphorus antisite $\mathrm{P}_{\mathrm{B}}$, boron vacancy $V_{B}$ and phosphorus vacancy $V_{P}$ defects. The simulated $\mathrm{P} L_{2,3}$ and $\mathrm{B} K$-edge spectra for the four different point defects are shown in Fig. 3.

As a reference, the experimental spectrum of sample $\mathrm{S} 1$ is shown as orange or green solid line for the $\mathrm{P} L_{2,3}$ and B $K$-edge, respectively. The solid black line represents the spectrum obtained by averaging the computed $\mathrm{x}$-ray absorption spectra for every possible system with its excited atom located within a radius of $3.8 \AA$ of the point defect, which, depending on the specific defect and absorption edge, ranges between 12 and 17 atoms. For each defect, an individual atomic spectrum is also shown with a black dashed line, which corresponds to an atom closest to the defect, in the case of a vacancy defect, or of the defect atom itself in the case of an antisite defect. Note that, in the $\mathrm{P} L_{2,3}$ spectra the $\mathrm{V}_{\mathrm{P}}$ and $\mathrm{B}_{\mathrm{P}}$, and likewise in the $\mathrm{B} K$-edge spectra for the $\mathrm{V}_{\mathrm{B}}$ and $\mathrm{P}_{\mathrm{B}}$, these indiviual atomic defect spectra are not visible as they overlap directly with their corresponding average spectrum. In these specific cases, all atoms considered in the average, are symmetrically identical with respect to the defect, due to the radial symmetry of the crystal.

The boron vacancy introduces a defect state and corresponding defect transition in the boron $K$-edge at around $189 \mathrm{eV}$, as evidenced by the small pre-edge feature in Fig. 3 (e). The intensity of the feature is rather low since the closest boron atoms to the vacancy are approximately at a distance of $3.25 \AA$. The effect on the spectrum of the phosphorus atoms directly adjacent to the vacancy, is much larger due to the proximity to the defect, and a significant contribution to the pre-edge in the $\mathrm{P} L_{2,3}$ spectrum can be seen in Fig. $3(\mathrm{a})$. This pre-edge feature is not observed in the experimental $\mathrm{P} L_{2,3}$ spectra, and since the effects of boron vacancies on both edges are intrinsically connected and cannot be regarded independently from one another, the hypothesis of boron vacancies causing the broad absorption onset in the experimental B $K$-edge, has to be rejected.

The same conclusion can be drawn for the phosphorus antisite $\mathrm{P}_{\mathrm{B}}$, but for opposite reasons. As Fig. 3 (d) shows, the defect does not introduce any defect transitions in the $\mathrm{P} L_{2,3}$ edge, below the absorption edge found in experiment, that could disqualify it. However, it also fails to explain the broad absorption edge of the B $K$-edge, as can be seen in Fig. $3(\mathrm{~h})$. The defects $\mathrm{V}_{\mathrm{P}}$ and $\mathrm{B}_{\mathrm{P}}$ both introduce defect transitions in the $\mathrm{B} K$-edge, which in the case of $\mathrm{V}_{\mathrm{P}}$ leads to a particularly intense feature due to the proximity of four atoms directly adjacent to the defect. The $\mathrm{P} L_{2,3}$ edge is not affected in a way that would discount these defects, except for a slight pre-edge feature that occurs in the $\mathrm{P} L_{2,3}$ edge for the $\mathrm{V}_{\mathrm{P}}$ defect, however its distance in energy with respect to the experimental absorption edge is within the experimental uncertainty due to intrinsic and instrumental broadening.

In summary, only $\mathrm{V}_{\mathrm{P}}$ and $\mathrm{B}_{\mathrm{P}}$ defects are predicted to introduce defect transitions in the $\mathrm{B} K$-edge, at energies that overlap with the experimentally observed broad absorption pre-edge feature, while not introducing defect transitions in the $\mathrm{P} L_{2,3}$ spectrum that are not observed in experiment. However, the width of the defect transitions in the B $K$-edge are distinctively narrow compared to the broad absorption onset found in the experiment. Even a linear combination of the different defects and their corresponding defect features is unlikely to result in such a smooth broad absorption onset. Spectral broadening in the DFT-XCH method is artificially simulated by the uniform linewidth broadening of individual defect states at a fixed width of $0.2 \mathrm{eV}$, which is an empirical value. This value accurately models the broadening of various defect states in boron compounds, such as boron vacancies and oxygen substitutional defects in $h$ $\mathrm{BN}^{19}$, as well as phosphorus vacancies in the icosahedral boron phosphide $\mathrm{B}_{12} \mathrm{P}_{2}{ }^{20}$. Even though the defect spectroscopy was simulated at a temperature of $0 \mathrm{~K}$ and spectral broadening can in principle be influenced by thermal structural distortions induced by a finite temperature, we have verified that the broadening of these defect transitions reamins unchanged at a finite temperature of $300 \mathrm{~K}$. Since the defect states, associated with the structural defects, are single excited states located in the band gap, which are therefore strongly localized, they are insensitive to perturbations of the local atomic structure. It therefore seems unlikely that the broad absorption onset in the B $K$-edge can be explained by the presence of antisite and or vacancy point defects.

\section{B. X-ray absorption spectroscopy of different crystal phases}

Another potential explanantion for the inability of the perfect cubic crystal lattice to reproduce the experimentally determined absorption onset, is that the experimental sample contained multiple crystal phases. A closely related material to boron phosphide is boron nitride, which, in addition to its cubic phase, has at least one other stable phase; the hexagonal phase. The boron $K$ edge for both the cubic $(c-\mathrm{BN})$ and hexagonal $(h-\mathrm{BN})$ phase of boron nitride have been studied extensively in the literature and are well understood. The hexagonal phase has characteristic trigonal bonding, whereas the bonding in the cubic phase is tetragonal and therefore, given the sensitivity of XAS to local structure and chemistry, the x-ray absorption spectra for these two phases are distinct, as shown in Fig. 4 (a).

The trigonal bonding in $h$-BN [dashed blue line in Fig. 4 (a)] gives rise to a strong isolated pre-edge absorption feature at $192 \mathrm{eV}$, due to a highly localized excitonic state with $\pi^{*}$ character ${ }^{19}$, which is located below the absorption edge of the spectrum of $c$-BN, indicated by the solid blue line in the same figure. Due to the many parallels that can be drawn between boron nitride and boron phosphide, it is reasonable to hypothesize that the hexagonal boron phosphide phase will have similar absorption 


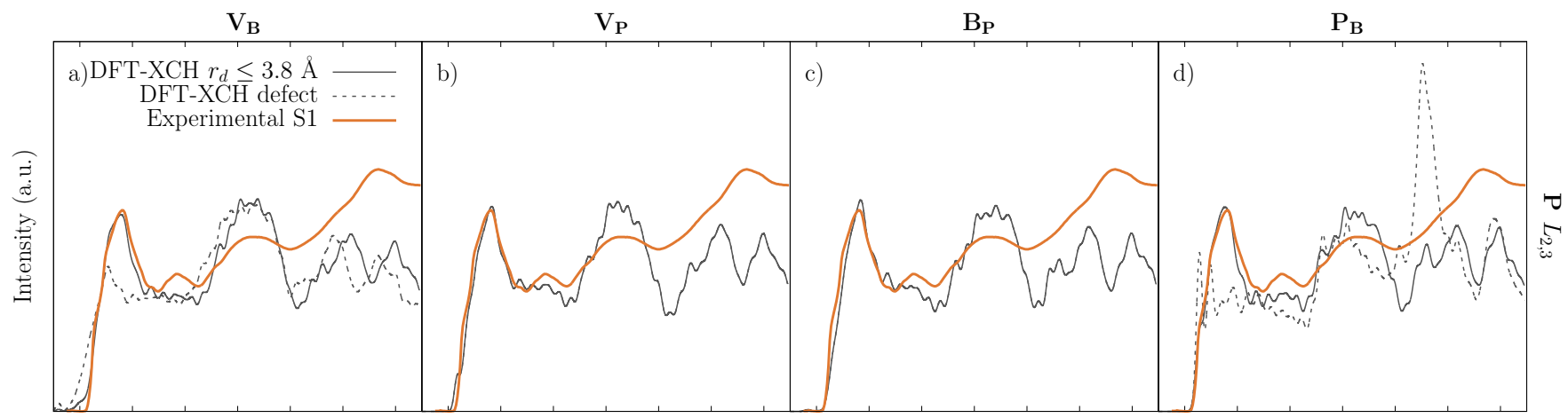

$\begin{array}{llllllllllllllllllllllllllll}130 & 134 & 138 & 142 & 146 & 150 & 154 & 130 & 134 & 138 & 142 & 146 & 150 & 154 & 130 & 134 & 138 & 142 & 146 & 150 & 154 & 130 & 134 & 138 & 142 & 146 & 150 & 154\end{array}$

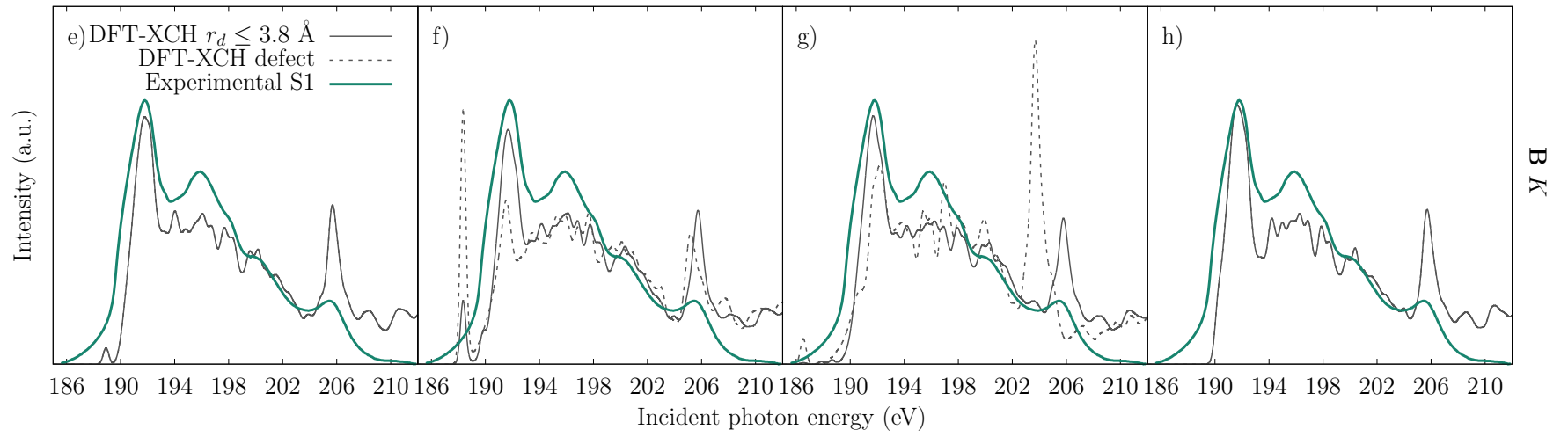

FIG. 3. Computed spectroscopic signatures in the $\mathrm{P} L_{2,3}$-edge [B $K$-edge] of a (a)[e] boron vacancy ( $\left.\mathrm{V}_{\mathrm{B}}\right)$, (b)[f] phosphorus vacancy $\left(\mathrm{V}_{\mathrm{B}}\right),(\mathrm{c})[\mathrm{g}]$ boron antisite $\left(\mathrm{B}_{\mathrm{P}}\right)$ and $(\mathrm{d})[\mathrm{h}]$ phosphorus antisite $\left(\mathrm{P}_{\mathrm{B}}\right)$ point defect in a $c$-BP crystal. The solid black line represents the spectrum obtained by averaging the computed x-ray absorption spectra for every possible system with its excited atom located within a radius of $3.8 \AA$ of the point defect. The dashed black line represents the computed spectrum for the excited system where the excited atom is the atom that constitutes the point defect or the atom directly adjacent to the defect.

spectroscopy characteristics and, like $h$-BN, will have a strong absorption feature below the main absorption edge of its tetragonally bonded cubic counterpart. Since $h$ $\mathrm{BP}$ has not yet been successfully synthesized in isolated form, no experimental data exists for its boron $K$-edge absorption spectrum, however, it can be predicted employing the DFT-XCH approach, given the success of said method in simulating and characterizing the B $K$-edge of $h$-BN.

The computed spectrum of the boron $K$-edge for a perfect $h$-BP crystal is shown in Fig. $4(\mathrm{~b})$. It bears a strong resemblence to that of the $h$-BN crystal structure, as it also has a strong isolated $\pi^{*}$ feature, followed by a broader feature resulting from excitations into electronic states with $\sigma^{*}$ character, albeit it red-shifted by at least $2 \mathrm{eV}$ and contracted significantly, as evident in the reduced separation between the $\pi^{*}$ and $\sigma^{*}$ peaks. There is no experimental reference for $h$-BP to permit energy alignment of the computed spectrum and employing the same energy shift used in the alignment of $c$-BP may not necessarily be correct. The energy aligment scheme employed in this work ${ }^{34}$, is designed to reference spectra of different structures but of similar chemical nature, to a single relative energy scale. In theory, a single constant value can then be used to align the computed x-ray absorption spectra for all structural configurations with respect to experiment. However, this method does not account for the presence of differing self-interaction errors in the computed energies of the highest occupied electronic orbital in the total energy of the core-hole excited state of the system, which are used directly in the alignment scheme.

As an example, due to the highly localized character of the first excited state in $h$-BN compared to the first excited state in $c$-BN, the self-interaction error is much larger in the former and therefore, accurate relative alignment consistent with experiment requires two different absolute alignment energy shifts of $191.5 \mathrm{eV}$ and $190.7 \mathrm{eV}$, respectively. Even though it is currently not possible to determine the correct absolute energy shift for $h$-BP, it should be smaller than the shift required for $h$-BN, since the first core-excited state used in the relative alignment is much more localized for $h$-BN as compared to $h$-BP. The difference in localization of the highest occupied electronic orbital for a boron $1 s$ excited state of $h$-BN and $h$-BP, is visualized in Fig. 5 . Nevertheless, we use the same value of $\Delta E$ for $h$-BP as the one used for the alignment of the $h$-BN spectrum to experiment, which guarantess an upper limit for the required absolute energy shift in the $h$-BP spectrum. In other 


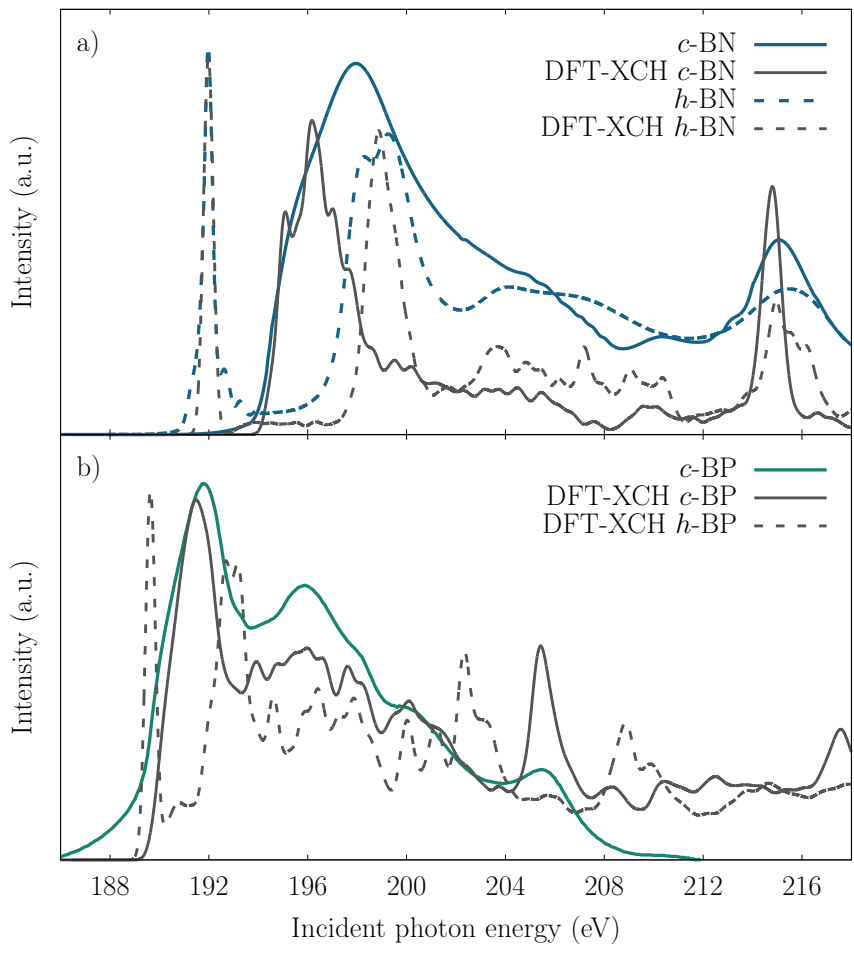

FIG. 4. Experimental and theoretical boron $K$-edge absorption spectroscopy of (a) boron nitride and (b) boron phosphide. The colored lines correspond to experimental data and the black lines are results obtained from calculations following the DFT-XCH method. Solid lines correspond to absorption spectra for cubic phases, whereas the dashed lines represent absorption spectra of hexagonal phases. The experimental data for the $h$-BN and $c$-BN phases were obtained from $\mathrm{Hu}-$ ber et al. ${ }^{19}$ and McCulloch et al. ${ }^{44}$, respectively.

words, we would expect the true $h$-BP boron $K$-edge to be slightly red-shifted with respect to the computed spectrum in Fig. 4 (b).

Under the uncertainties in absolute energy alignment in the computed $h$-BP spectrum, it is still possible that the $\pi^{*}$ excitation overlaps with the absorption onset of $c$-BP. However, the width of the $\pi^{*}$ feature is rather narrow and, reminiscent of the spectroscopic signatures of point defects discussed in Section V A, and such narrow spectroscopic features are unlikely to give rise to such a smooth broad absorption edge observed in the exprerimental XAS for $c$-BP.

A structural property that can affect the broadening of absorption spectral features is the degree of crystallinity. Even though x-ray absorption spectroscopy probes the local structure of a specific element and is therefore insensitive to long range order, a loss of crystallinity will also affect characteristic structural properties on a local scale. Amorphous phases may still predominantly possess a local bond order that resembles that of the perfect crystal, however the disruption of the long range order will cause typical values of structural properties, such as bond lengths and bond angles, to obtain wider distribu- tions. Spectral absorption features that directly correlate with these structural quantities will therefore experience a similar broadening.

\section{X-ray absorption spectroscopy of amorphous phases}

Once again, a suitable example to demonstrate the effect of amorphization of a material on its x-ray absorption spectroscopy, is hexagonal boron nitride. As a reference, Fig. 6 (a) depicts the B $K$-edge spectrum of the polycrystalline $h$-BN sample, that is also shown in Fig. 4 (a) and discussed in detail in the corresponding section. Figure 6 (b) depicts the B $K$-edge x-ray absorption spectrum collected in TEY mode for a boron nitride sample deposited with electron-beam evaporative physical vapor deposition (see Section II B for deposition details). Due to the low temperature during deposition, the structural character of this stochiometric boron nitride sample, is characterized not by crystalline but amorphous phases.

To model the absorption spectrum of the amorphous boron nitride sample, we have simulated an amorphous BN structure with a method implemented in ab initio molecular dynamics, details of which can be found in Section IIID. Starting from the perfect crystal, a liquid was generated by elevating the temperature above the melting point, allowing the system to diffuse. The melt was subsequently quenched and brought to room temperature, where the resulting amorphous network was equilibrated at a constant temperature of approximately $300 \mathrm{~K}$. From the equilibrated trajectory, multiple snapshots were taken, for which the x-ray absorption spectrum was calculated. An example of such a snapshot, taken from the amorphous boron nitride trajectory is shown in Fig. 7 (a). The average of the absorption spectra computed for all the configurational snapshots is plotted in Fig. 6 (b) as the solid green line. Comparing the experimental x-ray absorption spectra in Figs. 6 (a) and 6 (b), the changes in the boron $K$-edge, resulting from the amorphization of the hexagonal crystal structure, are captured well by the structural model of the amorphous boron nitride network. Overall, the characteristic spectral shape of the $h$-BN lattice is maintained, but the spectrum is significantly broadened. As mentioned before, this indicates that locally the trigonal bonding characteristic of the hexagonal lattice is maintained, but that typical bond lengths and angles are distorted from the optimal equilibrium values due to the absence of the long range order of a crystal lattice.

Structural differences between the crystal and amorphous configurations can be quantified by the radial distribution and bond angle distribution functions. The radial distribution function (RDF) of the simulated thermalized $h$-BN crystal structure and the amorphous BN network, shown in Figs. 6 (c) and 6 (d), respectively, represents the probability $g(r)$ of finding an atom at a distance $r$ from another atom. The dashed line considers 

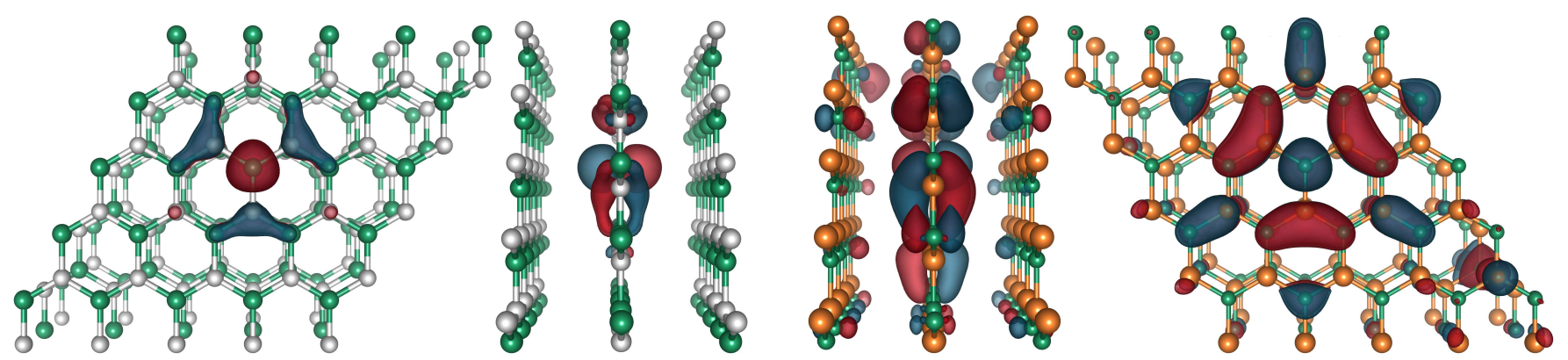

FIG. 5. From left to right, a front and side view of an isosurface of the squared wavefunction $|\psi(\vec{r})|^{2}$ of the highest occupied state for a boron $1 s$ excitation in $h$-BN and, in reverse order, in $h$-BP. The values of the isosurface drawn is chosen such that $50 \%$ of the total charge density norm is contained within the surface. The excited states in $h$-BN and $h$-BP both have a strong $\pi^{*}$ character, however the state in $h$-BN is clearly more localized compared to the same state in $h$-BP. The two different colors of the isosurfaces represent the phase of the wave function in that region.
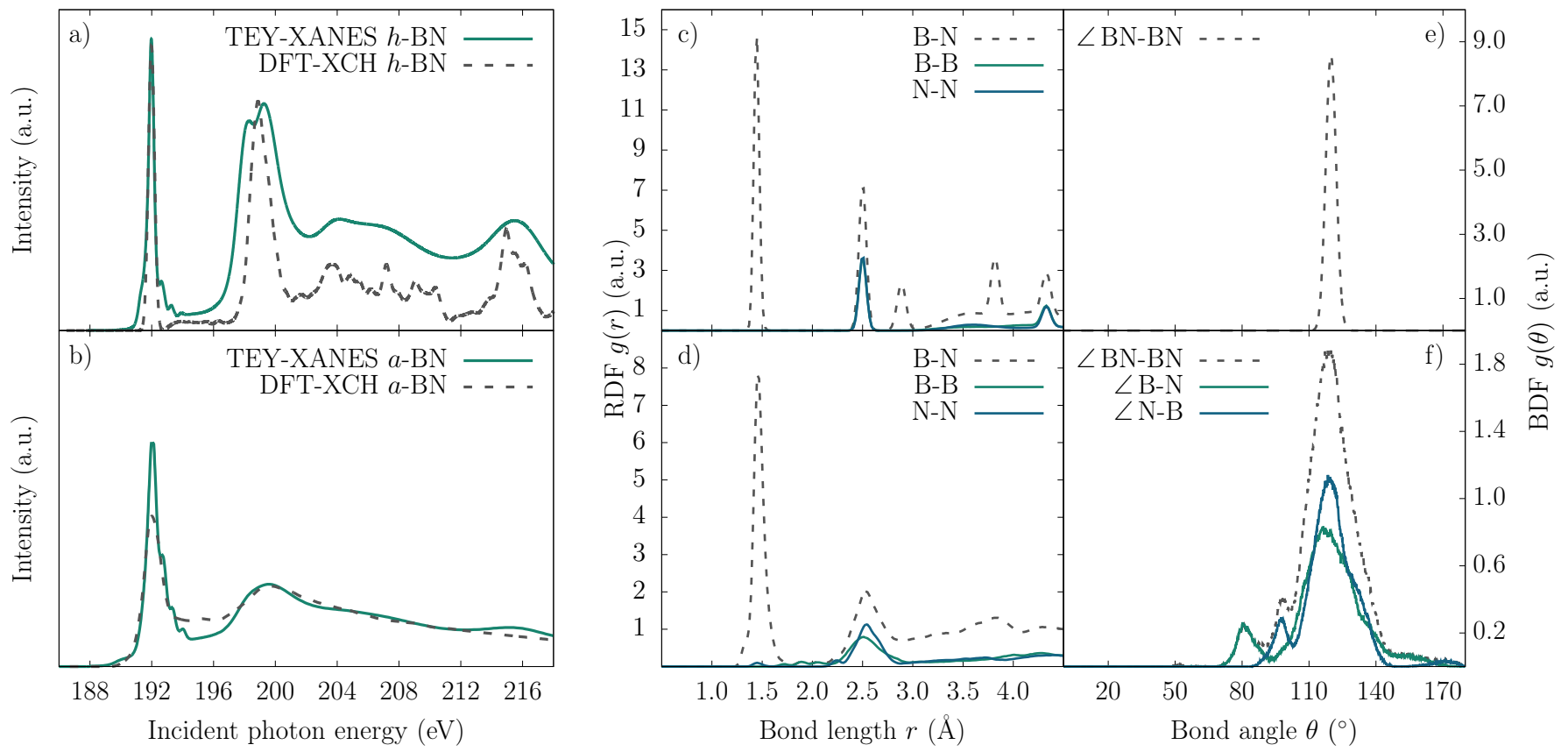

FIG. 6. Experimental and theoretical boron $K$-edge x-ray absorption spectra for (a) crystalline $(h$-BN) and (b) amorphous boron nitride $(a-\mathrm{BN})$. The solid green lines represent the experimentally collected XAS in TEY mode, whereas the dashed grey lines correspond to the computed spectrum for (a) the perfect crystal and (b) the amorphous network, respectively, both equilibrated around $300 \mathrm{~K}$. Panels (c) and (d) display the average radial distribution function for the equilibration trajectory of the crystal and amorphous structure, respectively. The dashed lines represent the distribution of the distance between both boron and nitrogen atoms, whereas the green and blue solid lines represent the homogeneous atomic distance distribution of boron and nitrogen atoms, respectively. Panels (e) and (f) display the average bond angle distribtution for the same molecular dynamics trajectories as described by the radial distribution function in panels (c) and (d). The dashed gray, solid green and solid blue line are calculated bond angle distributions for bond lengths up to $1.6 \AA$ and $1.8 \AA$ for figure (e) and (f), respectively.

both boron and nitrogen atoms and features a sharp peak around $1.46 \AA$ which is the characteristic bond length found in $h$-BN. The solid green and blue lines are defined similarly but only consider homogeneous boron or nitrogen bonds, respectively, and an absence of their contribution at the first-nearest neighbor peak in Fig. 6 (c), attests to the fact that there is no homogeneous bonding in the perfect crystal, as expected. For the amorphous structure, one can see a small contribution of homoge- neous bonding, with N-N bonds around $1.48 \AA$ and B-B bonds at a slightly larger typical bond length, however this is truely a small fraction of the entire sample. Compared to the crystal structure, the majority bonding in the amorphous network remains heterogeneous, however the typical bond lengths are distorted, as indicated by the broadening of the peaks in the radial distribution function.

The bond angle distribution function (BDF), which de- 

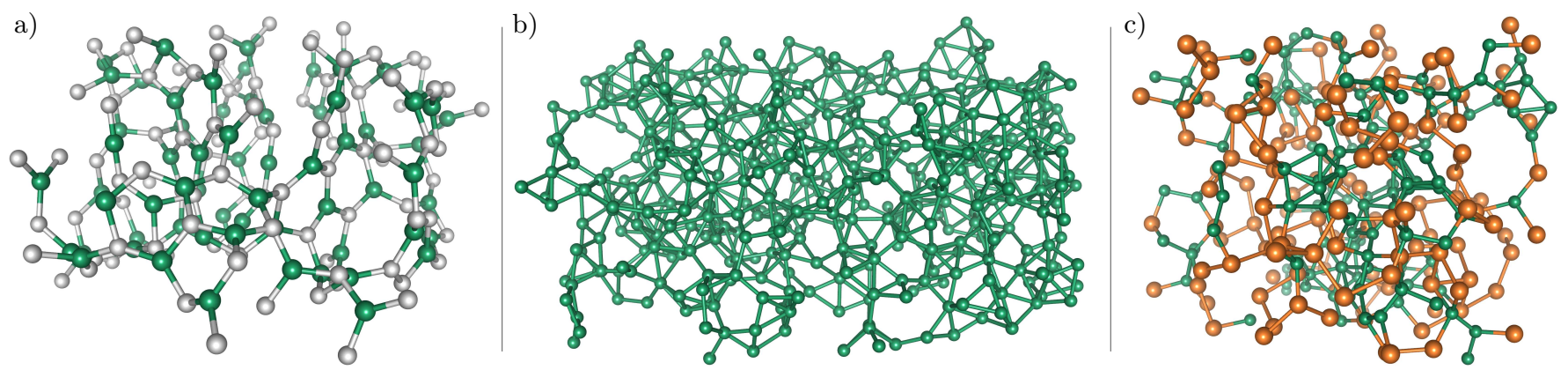

FIG. 7. Snapshots from a molecular dynamics trajectory of an (a) amorphous boron nitride, (b) amorphous boron and (c) amorphous boron phosphide structure, equilibrated at approximately $300 \mathrm{~K}$. Boron, nitrogen and phosphorus atoms are represented by green, gray and orange spheres, respectively. Boron-boron, boron-nitrogen and boron-phosphorus bonds are drawn up to maximum distance of $1.8,2.0$ and $2.4 \AA$, respectively.

fines the probability $g(\theta)$ of finding an atom with two bonds at an angle $\theta$, is plotted in Figs. 6 (e) and 6 (f), for the crystal and amorphous structure, respectively. The notation $\angle \mathrm{X}-\mathrm{Y}$, is defined as an angle formed by two bonds where the atom at the vertex is of type $\mathrm{X}$ and the atoms it is bound to are of type $\mathrm{Y}$. The types $\mathrm{X}$ and $\mathrm{Y}$ can each be a set of one or more elements. The $\mathrm{BDF}$ of the crystal structure is defined by a single peak at $120^{\circ}$ which is directly determined by the hexagonal symmetry of the hexagonal boron nitride lattice. Due to atomic vibrations, induced by the thermal effects of a finite temperature, which slightly displace atoms from their ideal equilibrium lattice positions, the distribution is not a delta function at this angle, but rather a slightly broadened distribution. The distribution of bond angles between all atoms, as found in the amorphous network, is significantly broader, but is still centered around an angle of $120^{\circ}$, characteristic of trigonal bonding. The two satellite peaks centered around a bond angle of $80^{\circ}$ and $100^{\circ}$ approximately, correspond to bond angles formed by heterogeneous bonds with a boron $(\angle \mathrm{B}-\mathrm{N})$ or nitrogen $(\angle \mathrm{N}-\mathrm{B})$ atom at the vertex, respectively, as is visualized by the solid green and blue line in Fig. 6 (f). In summary, the loss of long range order due to the amorphization of a crystal lattice, can significantly broaden spectral features in the x-ray absorption spectroscopy, however the overall spectral character will still be determined by the local chemistry and structural order of the element that is being excited.

To determine whether amorphous boron phosphide phases could explain the broad absorption onset observed in the experimental B $K$-edge spectra collected for $c$-BP samples, an amorphous BP network ( $a$-BP) was simulated in similar fashion as the amorphous boron nitride structure, described previously. A $c$-BP supercell was melted to create a liquid, which was subsequently quenched, resulting in an amorphous structure, a snapshot of which is visualized in Fig. 7 (c). In stark contrast with boron nitride, in the boron phosphide melt and its derived quenched amorphous configurations, a strong segregation of boron and phosphorous atoms was

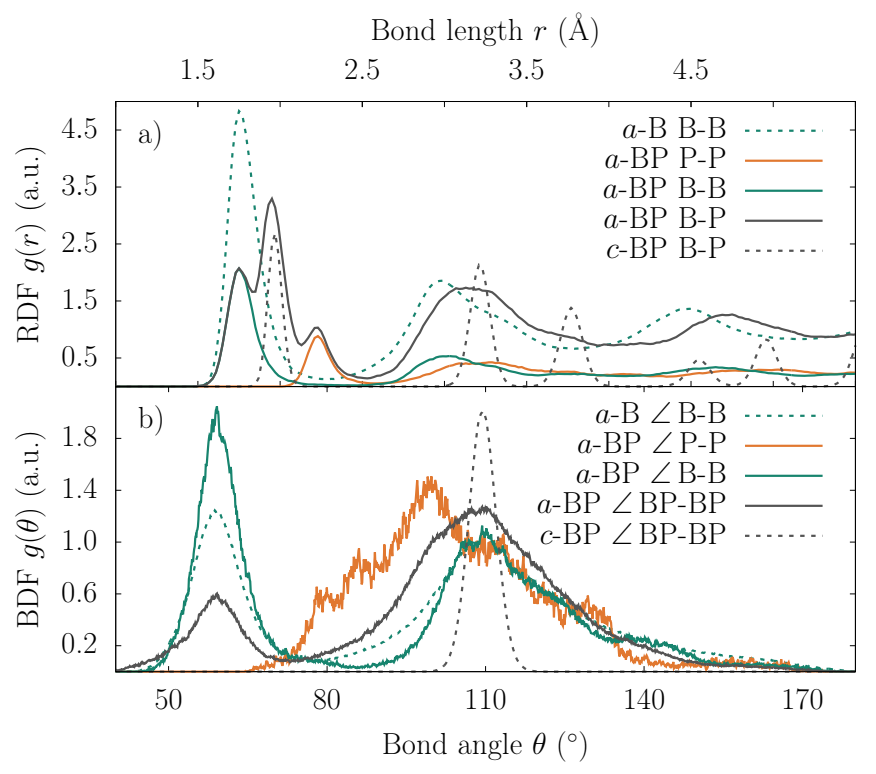

FIG. 8. The (a) radial distribution an (b) bond angle distribution function for the simulated amorphous boron phosphide network, calculated as an average over 9000 snapshots from the trajectory of the structure equilibrated at room temperature. The solid black lines correspond to bonds and bond angles between all boron and phosphorus atoms, whereas the solid orange and green line only consider homogenous bonds between phosphorous and boron atoms, respectively. The dashed green and black lines in the top (bottom) panel corresponds to the computed RDF (BDF) of an amorphous boron and cubic boron phosphide structure, and serve as a reference.

observed. Almost instantaneously following the nucleation point of the melting process, boron atoms were observed to group together, leading to separate boron and phosphorous clusters. This observation can be quantified by the average RDF and BDF for the amorphous boron phosphide trajectory equilibrated at room temperature, as plotted in Figs. 8 (a) and 8 (b), respectively.

The computed radial distribution functions for the boron nitride structures, both crystalline and amorphous 
as shown in Figs. $6(\mathrm{c})$ and $6(\mathrm{~d})$, showed a first-nearest neighbor peak that was clearly defined by a single Gaussian distribution, indicating that all atomic bonds belonged to a single population type and had similar character. Contrastingly, the first-nearest neighbor peak in the RDF computed for the amorphous boron phosphide trajectory, clearly comprises at least three distinct populations of bond lengths, as evidenced by the solid black line in Fig. 8 (a). The position of the highest peak out of the three overlaps with the position of the RDF for the perfect $c$-BP crystal thermally equilibrated at room temperature, represented by the dashed black line, indicating that there is still a significant population of boronphosphorus bonds with a bond length comparable to the one found in the cubic crystal. The deconvoluted distribution, represented partly by the green (orange) solid curve in the same figure, shows that the shorter (longer) bond lengths can be attributed to homogeneous boron (phosphorous) bonds. This is a direct result of the earlier described segregation of boron and phosphorous atoms in separate clusters, directly following the melting of the $c$ BP crystal.

A similar effect can be seen in the bond angle distribution function computed for the amorphous boron phosphide structure, which is plotted in Fig. $8(\mathrm{~b})$. The $\mathrm{BDF}$ of the $a$-BP structure still has a majority contribution around an angle of $109^{\circ}$, which is characteristic of tetragonal bonding, as evidenced by the BDF computed for the perfect $c$-BP crystal, represented by the dashed black line. However, the bond angle distribution for $a$ $\mathrm{BP}$ is considerably broadened and a new feature at $60^{\circ}$ appears which does not correspond to tetragonal bonding found in $c$-BP. The deconvolution of the BDF in the distribution function of homogeneously bonded atoms, reveals that the contribution at $60^{\circ}$ can be attributed to homogeneous boron bonding, as evidenced by the solid green line. This is again due to the presence of local boron clusters in the amorphous boron phosphide structure, in which the boron atoms predominantly form rings amongst each other in the shape of equilateral triangles.

Quantifying the sizes and distributions of rings formed in amorphous networks is another useful measure to describe non-crystalline systems. We have employed the shortest-path ring analysis as described by Franzblau ${ }^{45}$, which finds all the shortest-path rings in a structural network and determines their ring length distribution. A ring is defined as any closed path formed by neighboring atoms at a pre-defined maximum distance. With this simple definition, rings of arbitrarily large size can be found in almost any network, reducing the descriptive value of the structural property, which is why the constraint of shortest-path is added to the definition. This addition requires that any path does not contain a shorter closed path within itself. With this definition, we have computed the averaged shortest-ring path distributions for the trajectories of the amorphous boron, boron nitride and boron phosphide systems, as shown in Fig. 9. The amorphous boron nitride network has shortest-path

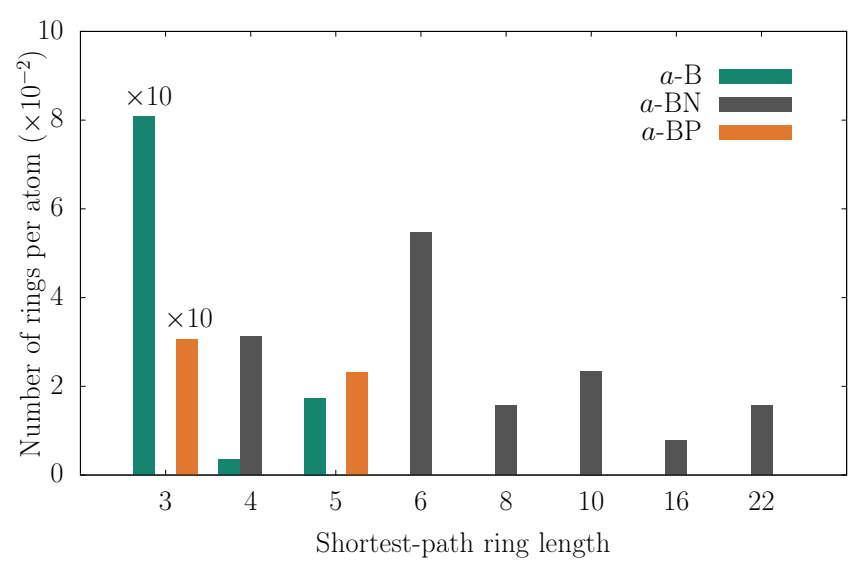

FIG. 9. Shortest-ring path analysis performed on the equilibrated trajectories of the amorphous boron $(a-\mathrm{B})$, boron nitride $(a$-BN) and boron phosphide ( $a$-BP) networks. The horizontal axis defines the size of the ring, or number of atoms that make up the ring, and the vertical axis is the frequency of occurrence of that ring size. All frequencies are normalized to the number of atoms in the cell of the simulation. The frequencies for rings of size 3 have been scaled down by a factor of 10 to improve visual clarity.

rings of varying lengths, but all of them contain an even number of atoms. This is a direct result of the predominantly heterogeneous bonding of this amorphous structure, as evidenced by the previously discussed RDF and BDF shown in Fig. 6. If there is no homogeneous bonding, then any shortest-path ring has to consist of an even numbers of atoms. The most populous ring type in amorphous boron nitride is made of 6 atoms, which corresponds directly to the hexagonal rings of the $h$-BN crystal structure which shows once more that, despite the lack of long range order, the amorphous network maintains a local structure that is reminiscent of the hexagonal crystal lattice. The amorphous boron network does not contain any rings longer than 5 atoms and rings formed by three boron atoms are numerous. These rings of 3 boron atoms take on the shape of equilateral triangles, exactly as they are found in the icosahedral units of the icosahedral boron crystal structures. The shortest-path ring distribution for the amorphous boron phosphide network is highly comparable to that of the amorphous boron structure and confirms the similarities between the $a$-B and $a$-BP structures.

The similarity between $a$-B and $a$-BP networks, is corroborated by the RDF and BDF as shown in Figs. 8 (a) and $8(\mathrm{~b})$, where the dashed green line represents the computed RDF and BDF, respectively, for an amorphous boron structure equilibrated at room temperature. The characteristic bond length is centered around $1.76 \AA$ and the typical bond angles are $60^{\circ}$ and $107^{\circ}$, which correspond exactly to the dominant bond lengths and bond angles found in icosahedral borides. The typical bond lengths and angles of the B-B bonds in $a$-BP show a high degree of similarity with the characteristic values found 


\begin{tabular}{lccc}
\hline Phase & $N_{\text {atom }}$ cell $^{-1}$ & $E_{\text {tot }}$ & $\Delta H_{f}$ \\
\hline$\alpha$-B & 36 & -241.2 & -12.7 \\
$c$-BP & 8 & -51.7 & -10.7 \\
$h$-BP & 4 & -24.3 & -9.9 \\
\hline$h$-BN & 4 & -35.1 & -14.1 \\
$c-\mathrm{BN}$ & 8 & -70.0 & -9.5 \\
\hline
\end{tabular}

TABLE III. Computed formation enthalpies for different boride crystal phases. The second column represents the number of atoms in the unit cell used in the calculation, $E_{\text {tot }}$ represents the total energy for the unit cell as obtained through DFT and $\Delta H_{f}$ is the derived formation enthalpy from its atomic constituents per formula unit. Both the total energy and formation enthalpy are given in $\mathrm{eV}$.

in the simulated amorphous boron structure, so it is apt to label the boron clusters in the $a$-BP as an amorphous boron phase. This raises the question, why boron nitride maintains a predominantly heterogeneously and trigonally bonded structure when amorphisized by quenching from a melt, yet in boron phoshide, a phase separation is observed, where its atomic constituents group into elementally homogeneous clusters and the boron atoms seem to form structures characteristic of purely amorphous boron phases. To answer this question, one can approach the formation of the various boron compounds discussed so far, from a thermodynamic point of view.

\section{Formation enthalpies of boron compounds}

The stability and likelihood of formation of a certain crystal phase can be estimated by calculating the formation enthalpy. To assess the probability of hexagonal boron phosphide or amorphous boron phases growing in parallel with the intended cubic phase of the studied boron phosphide samples, we have computed the formation enthalpies of various boron and boron phosphide phases, as tabulated in Table III.

The formation enthalpy $\Delta H_{f}$ in Table III is given per formula unit and is computed as

$$
\Delta H_{f}=E_{\mathrm{tot}}-\sum_{i}^{N} n_{i} E_{i}
$$

where $E_{\text {tot }}$ is the total energy of the system and the sum iterates over all $N$ elemental types $X$ in the cell, where $n_{i}$ corresponds to the number of atoms of type $X$ in the cell and $E_{i}$ represents the total energy of an isolated atom of type $X$.

The lower the formation enthalpy, the more stable and the more likely the corresponding crystal phase is to form. The computed $\Delta H_{f}$ show that the cubic phase of boron phosphide is more stable compared to the hexagonal phase, which is in line with the successful synthesiza- tion of $c$-BP but the current lack of a pure $h$-BP synthesis process. We have also computed the formation enthalpy of the $\alpha$-rhombohedral phase of elemental boron, labeled $\alpha$-B in Table III, which is energetically more favorable to form than both boron phosphide phases. This result is in line with the observation described in the previous section, that upon melting of boron phosphide, the boron and phosphorus atoms tend to segregate into homogeneous clusters, where the boron atoms form structures with structural properties characterstic for icosahedral boron phases. Consequently, this suggests that in a boron phosphide growing process, there is a reasonable chance of the formation of elemental boron phases. Note that contrastingly, hexagonal boron nitride is energetically more favorable than the elemental boride structure, as was already suggested by the lack of homogeneous bonding found in both the experimental and theoretical analyses of amorphous boron nitride. We now pose the question, whether the presence of amorphous boron phases in the experimentally produced $c$-BP samples, could explain the broad absorption onset observed in the experimental boron $K$-edge absorption spectrum.

\section{E. X-ray absorption spectroscopy of amorphous boron}

We have collected the B $K$-edge for an amorphous boron sample, deposited with electron beam evaporated physical vapor deposition, represented by the solid green line in Fig. 10. Details of the deposition can be found in Section IIB. The solid black line in the same figure is the calculated spectrum for the simulated amorphous boron structure and the dashed green line references the experimentally collected spectrum for the $c$-BP sample S1.

For the amorphous boron structures, both experiment and theory find a broad and smooth spectrum without any distinctive narrow spectral features. The dashed line labeled $C$, marks the position of a spectral feature present in the experimental spectrum at $194 \mathrm{eV}$, due to the boron oxide $\mathrm{B}_{2} \mathrm{O}_{3}$ phase ${ }^{19}$, which is present on the sample surface due to exposure to atmospheric conditions after sample deposition. Most interestingly, both the simulated and experimental spectrum, show a broad and gradual onset of the absorption edge, reminiscent of what has been observed for the $c$-BP samples investigated in this work, shown as a dashed green line in Fig. 10. To show that the broad onset is indeed caused by the amorphous character of the boride, we have also simulated the boron $K$-edge for the most simple allotrope of elemental boron, $\alpha$-rhombohedral boron $(\alpha-\mathrm{B})$, which is represented by the dashed black line in Fig. 10. The most prominent feature in the $\alpha$-rhombohedral spectrum, indicated by the dashed line labeled $B$, by definition associated with icosahedral boron atoms, is also still present in the amorphous boron spectrum, which proves that there are boron atoms present in the amorphous structure with 
$\begin{array}{lll}A & B & C\end{array}$

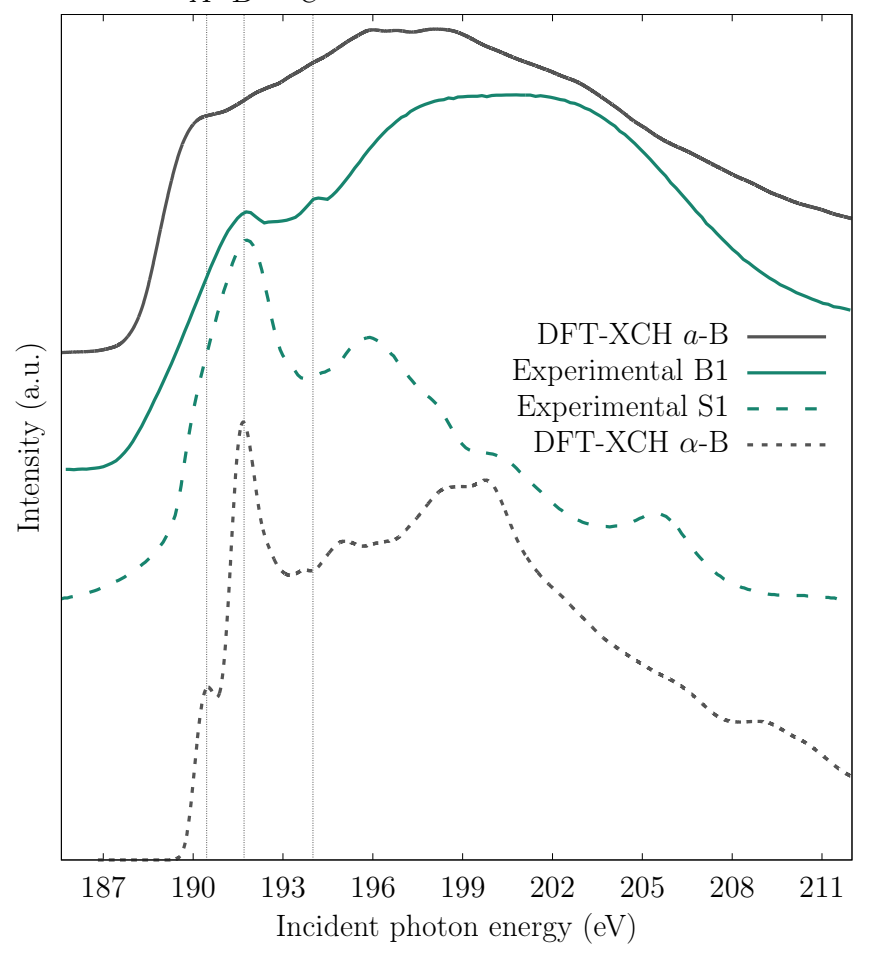

FIG. 10. Experimentally collected x-ray absorption spectra of an amorphous boron (B1) and cubic boron phosphide (S1) sample, represented by a solid and dashed green line, respectively. The solid and dashed black lines represent the simulated B $K$-edge spectrum for the amorphous boron structure and the thermally equilibrated $\alpha$-rhombohedral boron crystal structure, respectively. The spectra have been translated vertically in order to improve visual clarity.

an icosahedral configuration. However, the spectrum for the rhombohedral boron crystal structure clearly has a sharper absorption onset and is significantly blue shifted compared to the other spectra. This shows that it is not the crystalline but the amorphous character of the boron structure, that is responsible for the broad and gradual absorpion onset. This gives reasonable cause to believe that the broad absorption edge, as observed for $c$ - $\mathrm{BP}$, is due to the presence of amorphous boron phases in the studied samples.

Moreover, this hypothesis concerns a structural model that, containing exclusively boron atoms, strictly only influences the spectrum for excitations of $1 s$ electrons from boron atoms (i.e. the boron $K$-edge). By adding amorphous boron phases, to the initial purely cubic boron phosphide crystalline structural model of the $c$-BP samples, only the boron $K$-edge spectroscopy is affected and the phosphorus $L_{2,3}$ spectrum remains unchanged. The hypothesis of the presence of amorphous boron phases in the $c$-BP samples, therefore also explains the juxtaposition of the ability of the cubic boron phosphide structure to successfully model the $\mathrm{P} L_{2,3}$-edge and its simultaneous inability to accurately reproduce the $\mathrm{B} K$-edge. It also indirectly implies that the boron to phosphorus ratio in the samples has to be off-stoichiometric, with an excess of boron.

The XPS analysis presented in Section IV A has already shown that the samples are in fact nonstoichiometric and have an excess of boron. It is possible that this excess of boron is concentrated in amorphous boron phases, but the XRD and SEM data (see Supplementary Information) can not definitely prove this hypothesis. However, given that both samples are definitely not perfectly crystalline, the presence of amorphous phases, for example at the grain boundaries, is certainly not unlikely.

At the soft x-ray energies of the $\mathrm{P} L_{2,3^{-}}$and $\mathrm{B} K$ edge, the TEY operation mode of XANES is surface sensitive, typically on the order of a few $\mathrm{nm}^{46}$. The dominant relaxation mechanism for excited electrons at these photon energies operates through the emission of Auger electrons, which repeatedly scatter inelastically and in turn dominate the current that is measured in the experiment. The typical inelastic mean free path of these secondary electrons is not known for boron phosphide, but for comparable inorganic materials it is of the order of several $\AA^{47}$. This limited mean free path results in an approximate maximum probing depth of 3 to $4 \mathrm{~nm}$, for both the $\mathrm{P} L_{2,3^{-}}$and $\mathrm{B} K$-edge ${ }^{46}$. Therefore, all the presented statements on the structure of the $c$-BP samples based on x-ray absorption spectroscopy, can only be considered to apply to the top few $\mathrm{nm}$ of the samples. XPS is similarly surface sensitive and therefore the same caveat applies to those results as well.

\section{CONCLUSIONS}

We have presented experimentally collected x-ray absorption near-edge spectroscopy measurements at the boron $K$-edge and phosphorus $L_{2,3}$-edge for cubic boron phosphide samples, grown with chemical vapor deposition. Simulated x-ray absorption spectra from first principles show that the $\mathrm{P} L_{2,3}$-edge is aptly described by a simple $c$-BP crystal structural model, however the same model fails to reproduce the broad absorption onset observed in the B $K$-edge. Intrinsic crystal point defects and the hexagonal boron phosphide phase are discussed as a potential explanation, however calculations show that the corresponding spectroscopic signatures, or a superposition of a combination of these structures are too narrow to be able to reproduce the smooth and broad character of the experimental B $K$-edge.

Broad spectral features can arise due to the amorphization of crystalline phases, which was illustrated by means of experimental and theoretical x-ray absorption spectra for hexagonal and amorphous boron nitride structures. $A b$ initio molecular dynamics simulations were employed to model amorphous boron phosphide structures, which revealed that upon the melting of a $\mathrm{BP}$ crystal, boron and phosphorous atoms segregated in predominantly ho- 
mogeneously bonded clusters. This effect was explained by the formation enthalpies of the various boride phases, which showed that the formation of an elemental boron phase is energetically more favorable compared to the cubic boron phosphide phase. This leads to the conclusion that during the $c$-BP growth process there is a significant probability of the formation of amorphous boron phases. The boron clusters that formed in the simulated amorphous boron phosphide structure, were shown to adopt a structure commonly found in icosahedral and amorphous boron structures. Measured and simulated x-ray absorption spectroscopy of amorphous boron structures showed a broad absorption onset in their B $K$-edge, directly comparable to that observed in the spectra obtained for the $c$-BP samples.

A hypothesis positing the presence of amorphous boron phases in the grown $c$-BP thin films in order to explain the observed XAS, indirectly predicts that the B:P ratio should be off-stoichiometric with an excess of boron, which was indeed confirmed experimentally by XPS measurements. XRD and SEM data of the samples are compatible with the presence of amorphous phases but can not definitively prove it. Nonetheless, these results show that the broad absorption onset, measured in the B $K$ edge of the CVD grown $c$-BP samples, can be attributed to amorphous boron phases, which provides strong evidence for the presence of elemental boron clusters in the $c$-BP samples.

\section{ACKNOWLEDGMENTS}

This work is supported by NanoNextNL, a micro and nanotechnology programme of the Dutch Government and 130 partners. We acknowledge the support of the Center for X-ray Optics of Lawrence Berkeley Laboratory and the Industrial Focus Group XUV Optics at the MESA+ Institute for Nanotechnology at the University of Twente, notably the partners ASML, Carl Zeiss SMT GmbH, and the Foundation FOM. All the computational work was performed at the Molecular Foundry which is supported by the Office of Science, Office of Basic Energy Sciences, of the United States Department of Energy under Contract No. DE-AC02-05CH11231. *mail@sphuber.net

1 B. Padavala, C. Frye, Z. Ding, R. Chen, M. Dudley, B. Raghothamachar, N. Khan, and J. Edgar, Solid State Sciences 47, 55 (2015).

2 B. Padavala, C. D. Frye, X. Wang, Z. Ding, R. Chen, M. Dudley, B. Raghothamachar, P. Lu, B. N. Flanders, and J. H. Edgar, Crystal Growth \& Design 16, 981 (2016).

${ }^{3}$ G. Li, J. K. Abbott, J. D. Brasfield, P. Liu, A. Dale, G. Duscher, P. D. Rack, and C. S. Feigerle, Applied Surface Science 327, 7 (2015).

${ }^{4}$ L. Shi, P. Li, W. Zhou, T. Wang, K. Chang, H. Zhang, T. Kako, G. Liu, and J. Ye, Nano Energy 28, 158 (2016).

${ }^{5}$ Y. Kumashiro, Journal of Materials Research 5, 2933 (1990).

${ }^{6}$ Y. Ino, S. Nishimura, M. Hirai, S. Matsumoto, and K. Terashima, Japanese Journal of Applied Physics 52, 031201 (2013).

7 A. N. Caruso, Journal of Physics: Condensed Matter 22, 443201 (2010).

${ }^{8}$ V. Medvedev, A. Yakshin, R. van de Kruijs, and F. Bijkerk, Optical Materials Express 5, 1450 (2015).

9 S. P. Huber, V. V. Medvedev, J. Meyer-Ilse, E. Gullikson, B. Padavala, J. H. Edgar, J. M. Sturm, R. W. E. van de Kruijs, D. Prendergast, and F. Bijkerk, Optical Materials Express 6, 3946 (2016).

10 Z. Jia, J. Zhu, C. Jiang, W. Shen, J. Han, and R. Chen, Applied Surface Science 258, 356 (2011).

11 W. Liu, H. Tu, H. Yang, S. Zhang, L. Yan, C. Huo, and X. Su, Journal of Alloys and Compounds 538, 169 (2012).

12 V. A. Mukhanov, P. S. Sokolov, Y. L. Godec, and V. L. Solozhenko, J. Superhard Mater. 35, 415 (2013).

13 V. A. Mukhanov, P. S. Sokolov, O. Brinza, D. Vrel, and V. L. Solozhenko, J. Superhard Mater. 36, 18 (2014).
${ }^{14}$ H. Sugimoto, M. Fujii, and K. Imakita, RSC Adv. 5, 8427 (2015).

15 V. A. Mukhanov, D. Vrel, P. S. Sokolov, Y. L. Godec, and V. L. Solozhenko, Dalton Trans. 45, 10122 (2016).

16 B. Padavala, C. Frye, X. Wang, B. Raghothamachar, and J. Edgar, Journal of Crystal Growth 449, 15 (2016).

17 K. Woo, K. Lee, and K. Kovnir, Materials Research Express 3, 074003 (2016).

18 I. Jiménez, A. F. Jankowski, L. J. Terminello, D. G. J. Sutherland, J. A. Carlisle, G. L. Doll, W. M. Tong, D. K. Shuh, and F. J. Himpsel, Phys. Rev. B 55, 12025 (1997).

19 S. P. Huber, E. Gullikson, R. W. E. van de Kruijs, F. Bijkerk, and D. Prendergast, Phys. Rev. B 92, 245310 (2015).

20 S. P. Huber, E. Gullikson, C. D. Frye, J. H. Edgar, R. W. E. van de Kruijs, F. Bijkerk, and D. Prendergast, In preparation (2016).

21 A. López-Castillo, Int. J. Quantum Chem. 112, 3152 (2012)

22 J. Yu and W. Guo, Appl. Phys. Lett. 106, 043107 (2015).

23 D. Çakır, D. Kecik, H. Sahin, E. Durgun, and F. M. Peeters, Phys. Chem. Chem. Phys. 17, 13013 (2015).

24 J. Dong, H. Li, and L. Li, NPG Asia Materials 5, e56 (2013)

25 M. Mirzaei and M. Meskinfam, Solid State Sciences 13, 1926 (2011).

26 Z. Sohbatzadeh, M. Roknabadi, N. Shahtahmasebi, and M. Behdani, Physica E: Low-dimensional Systems and Nanostructures 65, 61 (2015).

27 S. feng Wang and $\mathrm{X}$. jun $\mathrm{Wu}$, Chin. J. Chem. Phys. 28, 588 (2015).

28 J. Underwood and E. Gullikson, Journal of Electron Spectroscopy and Related Phenomena 92, 265 (1998).

29 E. M. Gullikson, S. Mrowka, and B. B. Kaufmann, "Recent developments in euv reflectometry at the advanced light 
source," (2001).

30 D. Prendergast and G. Galli, Phys. Rev. Lett. 96, 215502 (2006).

31 E. L. Shirley, Physical Review B 54, 16464 (1996).

32 D. Vanderbilt, Phys. Rev. B 41, 7892 (1990).

${ }^{33}$ K. Laasonen, R. Car, C. Lee, and D. Vanderbilt, Phys. Rev. B 43, 6796 (1991).

34 A. H. England, A. M. Duffin, C. P. Schwartz, J. S. Uejio, D. Prendergast, and R. J. Saykally, Chemical Physics Letters 514, 187 (2011).

${ }^{35}$ G. Kresse and J. Furthmüller, Physical Review B 54, 11169 (1996).

36 J. P. Perdew, K. Burke, and M. Ernzerhof, Phys. Rev. Lett. 77, 3865 (1996)

37 P. E. Blöchl, Physical Review B 50, 17953 (1994).

38 G. Kresse and D. Joubert, Physical Review B 59, 1758 (1999).

39 A. Tkatchenko and M. Scheffler, Phys. Rev. Lett. 102, 073005 (2009)
40 J. Scofield, Journal of Electron Spectroscopy and Related Phenomena 8, 129 (1976).

${ }^{41}$ M. Niibe, K. Miyamoto, T. Mitamura, and K. Mochiji, Journal of Vacuum Science \& Technology A 28, 1157 (2010).

42 I. Caretti and I. Jiménez, Journal of Applied Physics 110, 023511 (2011)

${ }^{43}$ K. Simonov, N. Vinogradov, M. Ng, A. Vinogradov, N. Mårtensson, and A. Preobrajenski, Surface Science 606, 564 (2012).

${ }^{44}$ D. McCulloch, D. Lau, R. Nicholls, and J. Perkins, Micron 43, 43 (2012).

45 D. S. Franzblau, Phys. Rev. B 44, 4925 (1991).

46 B. H. Frazer, B. Gilbert, B. R. Sonderegger, and G. D. Stasio, Surface Science 537, 161 (2003).

47 S. Tanuma, C. J. Powell, and D. R. Penn, Surface and Interface Analysis 21, 165 (1994). 